\title{
Small and Large Signal Analysis of Photonic Crystal Fano Laser
}

\section{Zali, Aref Rasoulzadeh; Moravvej-Farshi, Mohammad Kazem; Yu, Yi; Mørk, Jesper}

\section{Published in:}

Journal of Lightwave Technology

Link to article, DOI:

10.1109/JLT.2018.2877816

Publication date:

2018

Document Version

Peer reviewed version

Link back to DTU Orbit

Citation (APA):

Zali, A. R., Moravvej-Farshi, M. K., Yu, Y., \& Mørk, J. (2018). Small and Large Signal Analysis of Photonic Crystal Fano Laser. Journal of Lightwave Technology, 36(23), 5611 - 5616.

https://doi.org/10.1109/JLT.2018.2877816

\section{General rights}

Copyright and moral rights for the publications made accessible in the public portal are retained by the authors and/or other copyright owners and it is a condition of accessing publications that users recognise and abide by the legal requirements associated with these rights.

- Users may download and print one copy of any publication from the public portal for the purpose of private study or research.

- You may not further distribute the material or use it for any profit-making activity or commercial gain

- You may freely distribute the URL identifying the publication in the public portal

If you believe that this document breaches copyright please contact us providing details, and we will remove access to the work immediately and investigate your claim 


\title{
Small and Large Signal Analysis of Photonic Crystal Fano Laser
}

\author{
Aref Rasoulzadeh Zali ${ }^{1}$, Mohammad Kazem Moravvej-Farshi ${ }^{*}$, Senior Member, IEEE, Yi Yu ${ }^{2}$, and \\ Jesper Mork ${ }^{2}$
}

\begin{abstract}
We analyze the small- and large-signal response of a photonic crystal Fano laser (PhC-FL). Conventional current modulation, as well as modulation of the laser via the mirror, is investigated using a numerical approach as well as linear smallsignal analysis. The results show that the amplitude modulation bandwidth of one of the laser ports (the through-port) and the frequency modulation (FM) bandwidth of another port (the cross-port) extend to the THz region. Large-signal simulations of the laser response to a $500 \mathrm{Gbit} / \mathrm{s}$ pseudo-random bit sequence modulation of the nanocavity are in good agreement with the predictions of the small-signal analysis. Finally, it is investigated how the design of the Fano mirror, in particular, the qualityfactor $(Q)$ of the nanocavity, affects the modulation properties.
\end{abstract}

Index Terms - Fano Laser, Laser modulation, Laser dynamics, Fano resonance, Photonic Crystal Laser, Large-signal, Smallsignal

\section{INTRODUCTION}

In recent years, by introducing defects in photonic crystals (PhCs), high quality cavities and hence ultra-compact lasers have been realized [1-8]. Different types of PhC lasers have been realized, i.e., point-defect [3, 5], line-defect [6-9] and nano-beam [10] lasers, each with their advantages. Recently a different type of laser, a so-called Fano laser (FL), was suggested, where one of the laser mirrors is realized using a Fano interference between the continuum of waveguide modes and the discrete mode of a side-coupled nanocavity [11]. This Fano mirror only has a high reflectivity within a narrow bandwidth determined by the quality factor of the nanocavity and it was shown that the laser can be frequency modulated (FM) at frequencies largely exceeding the relaxation oscillation frequency by modulating the nanocavity resonance rather than the laser current $[11,12]$. Recently, the Fano laser was

Manuscript received -June 20, 2018; revised XXXX XX, 2018. First published XXX XX, 2018; current version published XXXX XX, 2018.

Financial supports from Tarbiat Modares University (grant \#IG-39703) and Villum Fonden (grant 8692) are acknowledged.

A. Rasoulzadeh Zali (aref.rasuolzadehzali@modares.ac.ir) and M. K. Moravvej-Farshi (moravvej@modares.ac.ir) are with the Nano Plasmo-Photonic Research Group, Faculty of Electrical and Computer Engineering, Tarbiat Modares University, P O Box 14115-194, Tehran 1411713116, Iran. Y. Yu (yiyu@fotonik.dtu.dk) and J. Mork (jesm@fotonik.dtu.dk) are with DTU Fotonik, Technical University of Denmark, DK-2800 Kongens Lyngby, Denmark.

*Corresponding author: moravvej@modares.ac.ir

Digital Object Identifier:

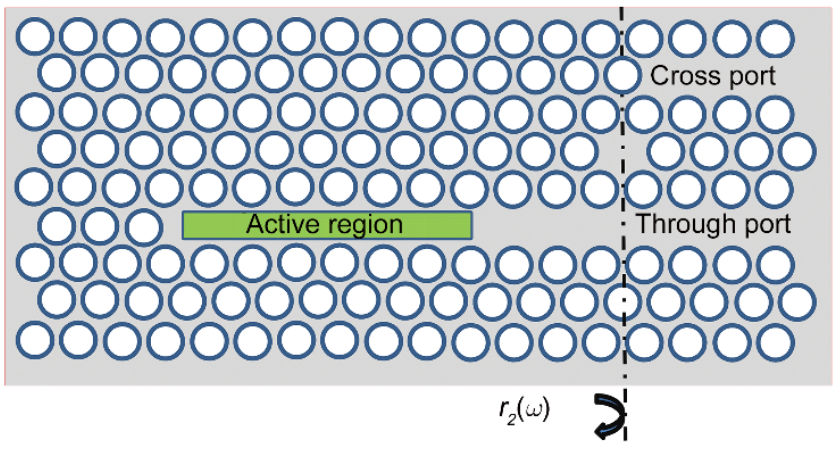

Fig. 1. Schematic of a photonic crystal Fano laser.

experimentally demonstrated [13] and it was furthermore shown that the laser can operate in a regime of self-pulsation, where a train of short pulses is generated [13,14].

In this paper, we extend the results of [11] by studying the large-signal modulation properties of the laser. The largesignal model is verified in the small-signal regime by comparison to semi-analytical small-signal results. Two different ways of modulating the Fano laser are investigated, i.e., via conventional current modulation or via modulation of the refractive index of the nanocavity, and the physical origin of the large difference in modulation bandwidth between the two schemes is explained. Both frequency modulation (FM) and amplitude modulation (AM) are being considered. Finally, the large signal response of the PhC-FL is investigated at bit-rates up to $500 \mathrm{Gbit} / \mathrm{s}$ by considering the response to a pseudorandom bit sequence. It is found that by proper choice of the output port of the Fano laser, both FM and AM modulation with good eye openings can be achieved at these extreme bitrates.

\section{MODEL AND STEADY-STATE CHARACTERISTICS}

A schematic of the PhC-FL under study is shown in Fig. 1. It is implemented in a III-V semiconductor membrane, where light confinement in the plane is due to the photonic crystal bandgap effect, realized by a pattern of airholes, and in the transverse direction light is confined by total internal reflection [15]. A line-defect $\mathrm{PhC}$ waveguide is terminated in one end by airholes while in the other end it is side-coupled to a point defect nanocavity giving rise to a Fano resonance [16]. The gain region is confined to the green region in the figure, 
Table I

Parameters used in simulations [11].

\begin{tabular}{cccc}
\hline Symbol & Quantity & Value & Unit \\
\hline $\mathrm{A}_{\text {Trans }}$ & Transverse waveguide area & 0.21 & $\mu \mathrm{m}^{2}$ \\
$\mathrm{~g}_{\mathrm{N}}$ & Differential Gain & $5 \times 10^{-20}$ & $\mathrm{~m}^{-2}$ \\
$L$ & Laser cavity length & 5 & $\mu \mathrm{m}$ \\
$n$ & Refractive index & 3.5 & \\
$N_{0}$ & Transparent carrier density & $10^{6}$ & $\mu^{-3}$ \\
$Q_{\mathrm{i}}$ & Intrinsic quality factor & $5 \times 10^{4}$ & \\
$Q_{\mathrm{pat}}$ & Quality factor associated with the & $1 \times 10^{4}$ & \\
$Q$ & cross-port & 500 & \\
$\mathrm{R}_{\mathrm{L}}$ & Total quality factor & 1 & \\
$\alpha$ & Left facet reflectivity & 1 & \\
$\alpha_{\mathrm{t}}$ & Linewidth enhancement factor & 10 & $\mathrm{~cm}^{-1}$ \\
$\Gamma$ & Internal absorption & 0.5 & \\
$\lambda_{\mathrm{r}}$ & Optical confinement factor & 1.55 & $\mu \mathrm{m}$ \\
$\tau_{\mathrm{N}}$ & Reference wavelength & 0.5 & $\mathrm{~ns}$ \\
\hline
\end{tabular}

i.e., the nanocavity is passive, which can be realized using the buried heterostructure technology [6]. The nanocavity is also coupled to an upper PhC waveguide, which is denoted as the laser cross-port, while the lower output waveguide is the through-port. It was shown in [11] that when the laser is operated at its minimum threshold gain, the power in the cross-port is much larger than that in the through-port, but bearing this in mind we shall here investigate the output properties of both ports. The bandwidth of the left mirror is very large and we shall for simplicity assume the reflectivity to be unity. The vertical dot-dashed line shows the horizontal position of the right mirror at which the nanocavity odd mode $\left(H_{1}\right)$ interferes destructively with the waveguide modes, resulting in a Fano mirror with effective reflection coefficient $r_{2}(\omega)=\gamma_{c} / \gamma_{T}\left(1+i \delta_{c}\right)$, in which $\delta_{c}=\left(\omega_{c}-\omega_{r}\right) / \gamma_{T}$ is the normalized nanocavity detuning, $\omega_{\mathrm{c}}$ is the nanocavity resonance frequency, and $\omega_{\mathrm{r}}=c / \lambda_{r}$ is the reference frequency about which the dispersive parameters are expanded [11-14]. Moreover, $\gamma_{c}$ and $\gamma_{T}=\gamma_{c}+\gamma_{i}+\gamma_{p} / 2$ represent the nanocavity-waveguide coupling rate and the total decay rate including the nanocavity intrinsic decay rate, $\gamma_{\mathrm{i}}$, and the decay rate into the cross-port, $\gamma_{\mathrm{p}}$ [11-14]. The corresponding quality factors are $Q_{x}=\omega_{r} / 2 \gamma_{x}$.

A dynamical model for the FL can be derived by combining a transmission line model and coupled-mode theory [11-14]. We use the same model as in [11], which is valid as long as the modulation of the nanocavity resonance is on the order of or smaller than the nanocavity linewidth [14] and the frequency is smaller than the inverse of the roundtrip time [12]. All the parameters used in our numerical simulations are listed in Table I.

The laser threshold current, $J_{\mathrm{th}}$, versus the detuning is shown in Fig. 2(a). When the nanocavity is detuned from its optimum value, the threshold current increases since the laser now oscillates off the center of the nanocavity resonance in order to fulfill the phase matching condition [11]. The threshold current is asymmetric with respect to detuning due to the finite value of the alpha parameter [11, 14]. Moreover, the laser output power emerging from the through- and cross-port are given by

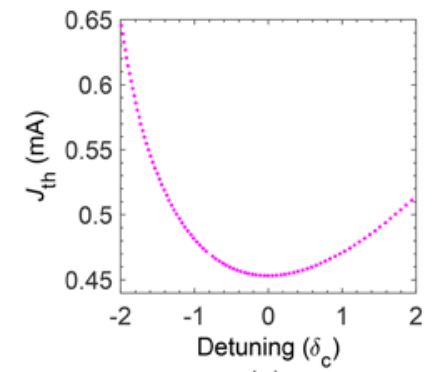

(a)

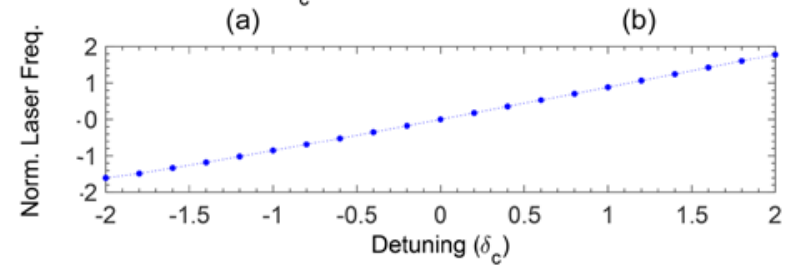

(c)

Fig. 2. (a) Threshold current versus nanocavity detuning. (b) Through-port and crossport powers versus nanocavity detuning, for constant bias currents of $4.5 \mathrm{~mA}$ (circles) and $6.8 \mathrm{~mA}$ (diamonds). (c) Laser frequency (nomalized to $\gamma_{\mathrm{T}}$ ) as a function of nanocavity detuning.

$P_{t}=2 \varepsilon_{0} n_{\text {eff }} c\left|A^{+}(t)-A^{-}(t)\right|^{2}$

and

$P_{c}=2 \varepsilon_{0} n_{\text {eff }} c \gamma_{p}\left|A^{-}(t)\right|^{2} / \gamma_{c}$,

respectively, in which $n_{\text {eff }}, \varepsilon_{0}$, and $c$, are the effective refractive index, free space permittivity, and light velocity. $A^{+}(t)=a^{+} \exp \left[j \phi^{+}(t)\right]$ and $A^{-}(t)=a^{-} \exp \left[j \phi^{-}(t)\right]$ represent the right- and left-propagating field envelopes at the reference plane adjacent to the Fano (right) mirror, with $a^{ \pm}$and $\phi^{ \pm}$being the corresponding amplitudes and phases. Fig. 2(b) illustrates the dependencies of $P_{\mathrm{t}}$ and $P_{\mathrm{c}}$ on the nanocavity detuning for two different bias currents of $4.5 \mathrm{~mA}$ and 6.8 $\mathrm{mA}$. For zero detuning, the laser frequency and the nanocavity resonance coincide, maximizing the destructive interference and hence minimizing the through-port power. The cross-port power is proportional to the optical energy stored in the nanocavity and is maximized for $\delta_{\mathrm{c}}=0$. As the nanocavity frequency is detuned, the power emerging from the throughport increases, corresponding to an increase of the differential quantum efficiency [11]. The corresponding variation of the laser frequency with detuning is shown in Fig. 2(c), displaying a quasi-linear dependence.

Next, we have analyzed the modulation properties of the laser using the standard small-signal (SMS) approach and compared the results with those obtained numerically. In the SMS approach, we used the first order perturbation for all the dynamical variables $\left(a^{+}, a^{-}\right.$, $\phi^{+}, \phi^{-}$, and carrier density, $N$ ) around the steady-state values [17].

\section{SMALL-SIGNAL MODULATION}

We consider two cases of modulation: (i) conventional current modulation defined by $J(t)=\Delta J \sin (2 \pi f t)$ with $\Delta J$ and $f$ being the modulation amplitude and frequency; (ii) modula- 


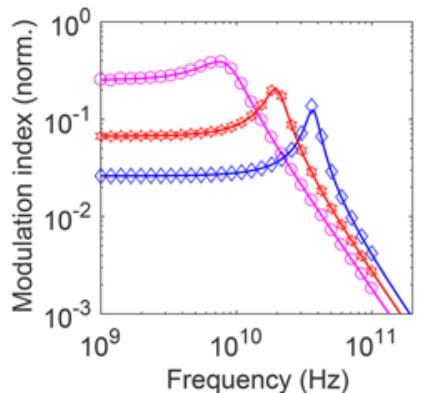

(a)

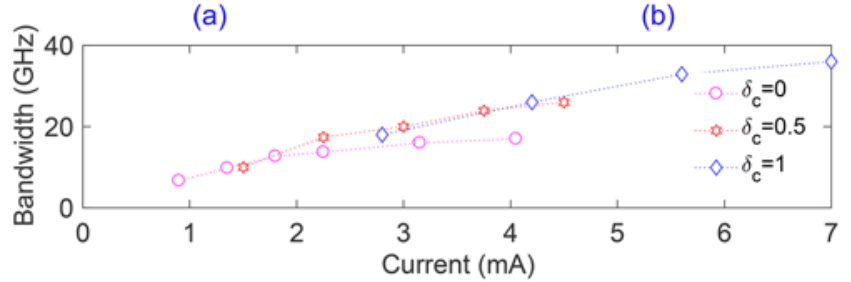

(c)

Fig. 3. Frequency response of the (a) power modulation index and (b) FM modulation amplitude of the PhC-FL for cross-port under current modulation with a fixed amplitude of $\Delta J=225 \mu \mathrm{A}$ and steady-state photon population of $1.6 \times 10^{4}$. The nanocavity detuning values are $\delta_{\mathrm{c}}=0$ (circles), 0.5 (hexagons), and 1 (diamonds), obtained via numerical approach. Data obtained via SMS approach are depicted by solid lines. (c) 3-dB bandwidth versus bias current for the three detuning values.

tion of the nanocavity frequency, defined by

$\Delta_{c}(t)=\gamma_{T}\left(\delta_{c}+\varepsilon \sin (2 \pi f t)\right)$

with $\varepsilon$ being the modulation amplitude.

For case (i), we consider a fixed amplitude of $\Delta J=225 \mu \mathrm{A}$ and compare the responses for three different (static) nanocavity detunings $\left(\delta_{\mathrm{c}}=0,0.5\right.$, and 1$)$, assuming the same steady state photon number of $1.6 \times 10^{4}$. Fig. 3(a) compares the modulation indices $\left(\Delta p_{x} / \bar{p}_{x}\right)$ i.e., the ratio of the maximum excursion of the optical power $\left(\Delta p_{\mathrm{x}}\right)$ to the average power at the cross-port $(x=c)$ or through-port $(x=t))$, calculated by numerical simulations (symbols) with the similar data obtained via the SMS approach (solid curves) for $\delta_{\mathrm{c}}=0$ (circles), 0.5 (hexagons), and 1 (diamonds). Fig. 3(b) shows a similar comparison for the FM amplitudes, defined as the maximum excursion of the instantaneous optical frequency $\left(v(t)=\partial_{t} \phi(t)\right)$. By increasing the detuning from $\delta_{\mathrm{c}}=0$ to 1 , the threshold current increases, so to keep the photon number constant $\left(1.6 \times 10^{4}\right)$, the laser bias current is increased, thus decreasing the ratio of the current modulation amplitude to the bias current. As a result, the low frequency component of the modulation index becomes smaller. Figs. 3(a) and (b) also reveal that the behavior of the FM amplitude and modulation index for the PhC-FLs in the low-frequency region is similar to the case of conventional FP lasers, where the modulation index is almost flat while the slope of the corresponding FM amplitude is large and positive for $\omega<\omega_{\mathrm{R}}$ [17]. Also, from these two figures, one can conclude that for the same intracavity photon number, the larger the Fano laser nanocavity detuning, the larger the relaxation oscillation frequency and bandwidth. For example, for the constant photon number of $1.6 \times 10^{4}$ and the given nanocavity detunings, the corresponding relaxation oscillation frequencies are $f_{\mathrm{R}}=9 \mathrm{GHz}$ (circles), $20 \mathrm{GHz}$ (hexagons), and $35 \mathrm{GHz}$ (dia-

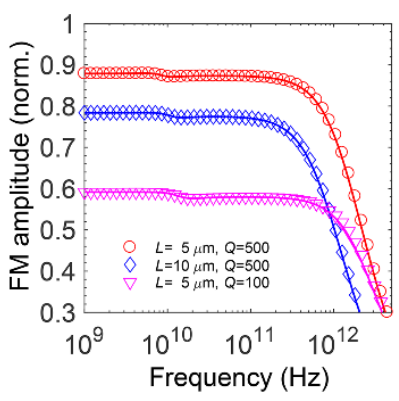

(a)

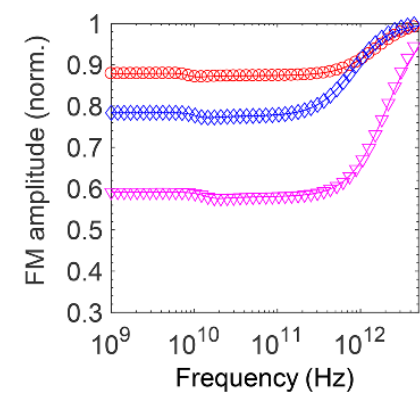

(b)
Fig. 4. FM amplitude of the (a) forward field $\left(A^{+}\right)$and (b) cross-port field $\left(A^{-}\right)$versus modulation frequency in a PhC-FL normalized to the modulation amplitude of the nanocavity detuning, $2 \varepsilon \gamma_{\mathrm{T}}$. The FLs have $Q=500$ and $L=5 \mu \mathrm{m}$ (circles), $Q=500$ and $L$ $=10 \mu \mathrm{m}$ (diamonds), $Q=100$ and $L=5 \mu \mathrm{m}$ (triangles), respectively. $\varepsilon$ is assumed to be 0.05 for all the curves.

monds). To further illustrate this, Fig. 3 (c) shows the PhC-FL 3 -dB bandwidth versus injection current for $\delta_{\mathrm{c}}=0,0.5$, and 1 . As for a conventional FP laser, by increasing the bias current, the 3-dB bandwidth of the FL increases. Here, we can see that the nanocavity detuning of the FL can also act as a possibility to increase the relaxation oscillation frequency and the associated 3-dB bandwidth, albeit, at the expense of greatly reducing the modulation efficiency. This is because as the detuning increases, the Fano mirror reflectivity decreases and so does the photon lifetime, which will increase the relaxation oscillation frequency [17].

As can be observed from Fig. 3(c), the nanolaser current injection level is in the range of $\mathrm{mA}$. This may raise an important issue regarding the impact of thermo-optical effects. Nonetheless, a thorough systematic thermal analysis, using A full 3-D simulation as well as experimental results, it has been shown that InP based PhC lasers remain at room temperature under CW pumping [18].

In the second case, we investigate the response of the FL to modulation of the nanocavity frequency. This may be implemented by various approaches: (i) Dynamic modulation of nanocavity resonance frequency by applying voltage to the electrodes placed near it [19]; (ii) Modifying the nanocavity resonance frequency, without changing its quality factor, by moving a near-field probe vertically and laterally in the nanocavity [20]; (iii) Modulation of the nanocavity resonance frequency, by means of optical nonlinearities - i.e., via dispersion of the optically excited free carriers [21, 22]. Varying the modulation frequency in the range of $1 \mathrm{GHz} \leq f \leq 30 \mathrm{THz}$, we calculate the FM amplitude. Fig. 4(a) and 4(b) show the frequency dependence of the FM amplitudes for $A^{+}$and $A^{-}$. Symbols represent the data obtained by the numerical approach and the solid curves depict the result of the SMS analysis. Circles in both figures represent the data for a PhC-FL with a nanocavity having a quality factor of $Q=500$ and a waveguide cavity having a length of $L=5 \mu \mathrm{m}$. Using the SMS approach based on our theoretical model, it can be found that the instantaneous optical frequency spectrum of the corresponding fields $\left(v^{+}(\omega)\right.$ and $\left.v^{-}(\omega)\right)$ can be approximated by 


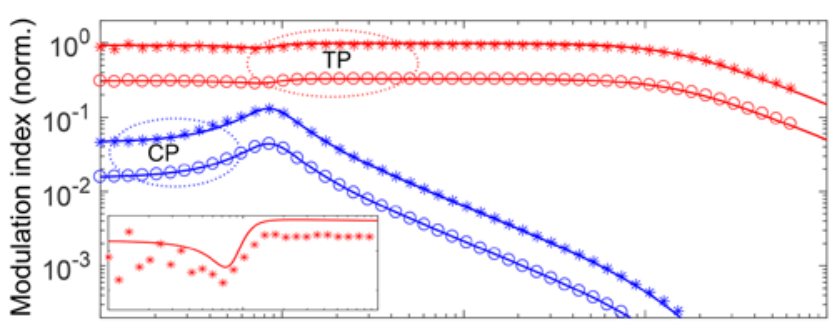

(a)

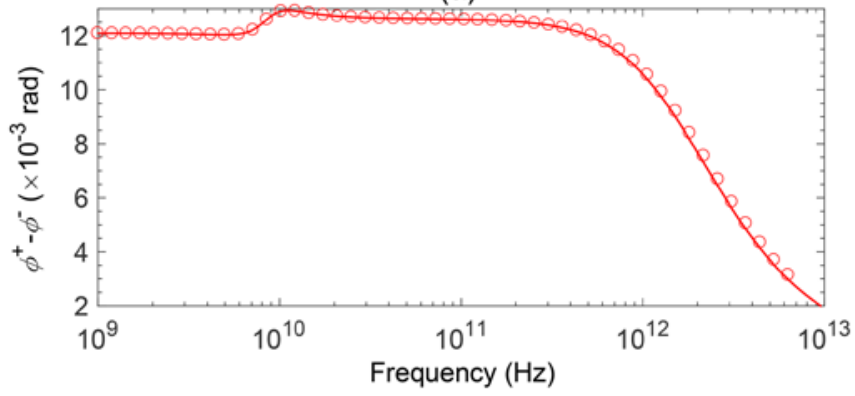

(b)

Fig. 5. Nanocavity modulation. (a) Frequency response of the intensity modulation index of the cross-port (blue) and through-port (red) power for $\varepsilon=0.05$ (circles) and $\varepsilon=0.25$ (crosses)., obtained via numerical approach. Here $J=5 J_{\text {th }}, Q=500$ and nanocavity detuning $\delta_{\mathrm{c}}=0.05$. (b) Phase difference between the counter-propagating fields within the line-defect cavity region of the FL for $\varepsilon=0.05$. Solid curves represent the corresponding data obtained via the SMS approach.

$v^{+}(\omega)=\frac{\gamma_{L}}{\left(\gamma_{L}+\gamma_{T}\right)} \frac{\left(1+i \omega /\left(\gamma_{L}+\gamma_{T}\right)\right)}{1+\left(i \omega /\left(\gamma_{L}+\gamma_{T}\right)\right)^{2}}$

and

$v^{-}(\omega)=\frac{\gamma_{L}}{\left(\gamma_{L}+\gamma_{T}\right)} \frac{\left(1+i \omega / \gamma_{L}\right)}{\left(1+i \omega /\left(\gamma_{L}+\gamma_{T}\right)\right)}$

as shown in Figs. 4(a) and (b). So the DC component of the FM amplitudes for both fields are $\gamma_{\mathrm{L}} /\left(\gamma_{\mathrm{T}}+\gamma_{\mathrm{L}}\right)$. As we can see from Fig. 4, for the PhC-FL with $Q=500\left(\gamma_{\mathrm{T}} / 2 \pi=194 \mathrm{GHz}\right)$ and $L=5 \mu \mathrm{m}\left(\gamma_{\mathrm{L}} / 2 \pi=1.34 \mathrm{THz}\right)$, the low frequency value of the FM amplitude is about 0.87, while, for the FL with $L=10 \mu \mathrm{m}$ and $Q=500$, the FM amplitude reduces to 0.78 , and for the FL with $Q=100$ and $L=5 \mu \mathrm{m}$, it decreases to 0.58 . As shown in [11], the cut-off frequency (bandwidth) of the FM response depends on the nanocavity decay rate $\left(\gamma_{\mathrm{T}}\right)$ and the inverse of the waveguide cavity round-trip time $\left(\gamma_{\mathrm{L}}\right)$, i.e., $\omega_{3 \mathrm{~dB}}=\left(\gamma_{\mathrm{T}}+\gamma_{\mathrm{L}}\right)$. Therefore, as $L$ increases, $\gamma_{\mathrm{L}}$ and hence the modulation bandwidth, for a given $Q$ decreases. Moreover, as the nanocavity quality factor decreases, the total decay rate increases and the FM response bandwidth increases. Fig. 4(b) shows that the FM amplitude increases towards unity with the modulation frequency, reflecting that the cross-port field modulation always tracks the nanocavity field change instantaneously. We notice that the model becomes inaccurate for high modulation frequencies exceeding the intermode-spacing [12], but this limit occurs at frequencies much higher than the relaxation oscillation resonance frequency, safely allowing analysis in the high-frequency regime not accessible in conventional lasers. In the low-frequency limit, the FM response represents the slope of the steady-state lasing frequency versus nanocavity resonance frequency. These results show that in contrast to the case of current modulation, SMS modulation of the Fano mirror resonance changes the phase and frequency of the field in the nanocavity via the adiabatic wavelength conversion mechanism that lets the laser field to track the nanocavity field, directly, leading to an essentially pure FM signal. This is because, the modulation frequency is much faster than carrier dynamics timescale that limits the conventional lasers bandwidths $[11,23]$. Consequently, the SMS analysis indicates that the usual bandwidth limitation imposed by relaxation oscillations in conventional current-modulated FP lasers is absent here [11].

Figure 5 (a) shows the frequency dependence of the modulation indices of the cross-port and through-port powers under an injection current of $J=5 J_{\text {th }}$ and modulation amplitudes of $\varepsilon=0.05$ (circles) and $\varepsilon=0.25$ (crosses). The numerical results (markers) agree quantitatively with the results of the SMS analysis. However, by increasing the modulation amplitude to $\varepsilon=0.25$, the deviation between the SMS and the numerical results starts to be apparent (see inset in Fig. 5(a)). The modulation spectra for the cross-port signals, with peaks observed around the relaxation oscillation frequencies, behave similarly to that of a conventional FP laser. Besides, as the modulation amplitude increases, the modulation index increases. Furthermore, for constant modulation amplitude, since the cross-port power is higher than that of the through-port, the intensity modulation index of the through-port power is larger.

As seen in Fig. 5(a), the intensity modulation bandwidth for the through-port power is in great contrast to that of the crossport. The bandwidth of the modulation index of the throughport power can be extended to the $\mathrm{THz}$ range. To understand this, let us compare the expressions for their corresponding small signal fluctuations. A simple calculation reveals that $d P c$ (small signal fluctuation of cross-port power) has no phase dependence (on $\phi^{+}$and/or $\phi^{-}$) whereas $d P_{\mathrm{t}}$ (small signal fluctuation of through-port power) is related to the phase difference of the counter-propagating signals through $\left(d \phi^{+}-d \phi^{-}\right) \sin \left(\phi^{+}-\phi^{-}\right)$, where $d \phi^{ \pm}$are small signal phase fluctuations. This dependence, flattens out the modulation index of the through-port power at high modulation frequencies, as can be seen from Fig. 5(b) where the spectrum $\left(d \phi^{+}-d \phi^{-}\right)$is almost flat up to $\sim 1 \mathrm{THz}$. In other words, the characteristic relaxation rate for the phase difference is governed by the total rate $\left(\gamma_{\mathrm{L}}+\gamma_{\mathrm{T}}\right)$ by which the phase coherence can decay, resulting in a $\mathrm{THz}$ bandwidth and circumventing the usual limitation imposed by the relaxation oscillation frequency [11].

\section{LARGE-SigNAL MODULATION}

Finally, to examine the capability of digital data transfer from electrical to the optical domain by the PhC-FL, we investigate the large signal behavior. In Ref. [11], it was shown that when the large-signal modulation amplitude exceeds a certain critical limit, short-pulses may be generated due to the build-up of a large carrier density during the part of the modulation cycle where the laser is below the threshold. In order to avoid these strong nonlinear effects, the modulation amplitude should be limited. Here we limit ourselves to positive detuning 

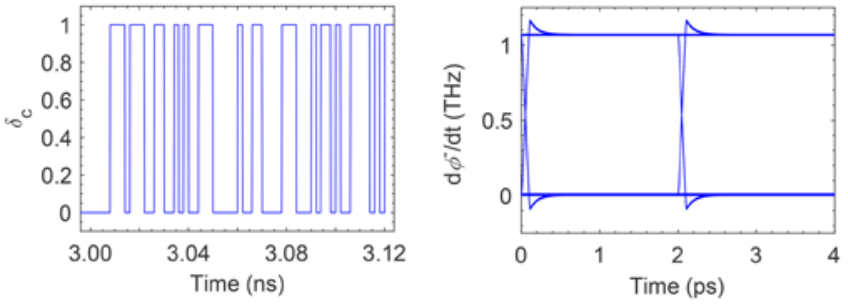

(a)

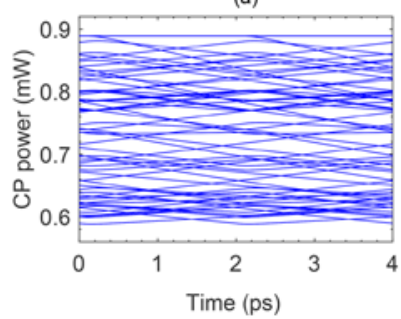

(c)

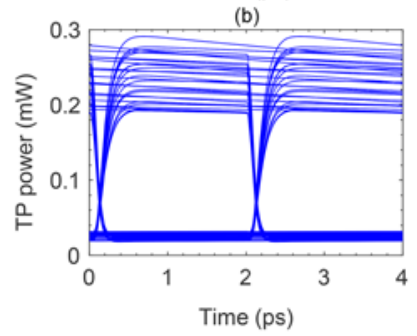

(d)

Fig. 6: Nanocavity modulation. (a) A 64-bit pseudo-random input data sequence used to modulate the nanocavity detuning at $500 \mathrm{~Gb} / \mathrm{s}$ with an amplitude of $0 \leq \delta_{\mathrm{c}}(\mathrm{t}) \leq 1$. Eye diagrams for the (b) cross-port FM amplitude, (c) cross-port power and (d) through-port power in a PhC-FL with $Q=500$ and $L=5 \mu \mathrm{m}$ for $J=5 J_{\text {th. }}$.

of the nanocavity and consider two cases, i.e., $0 \leq \delta_{\mathrm{c}}(\mathrm{t}) \leq 1$ and $0 \leq \delta_{\mathrm{c}}(\mathrm{t}) \leq 0.5$. We investigate the laser temporal responses under a pseudo-random binary sequence (PRBS) modulation of the nanocavity detuning, at $500 \mathrm{Gbit} / \mathrm{s}$, as illustrated in Fig. 6(a). This PRBS bit rate is chosen to be close to the 3-dB bandwidth of the SMS response of the FL. In order to evaluate the quality of the output modulation, we consider the corresponding eye diagrams. The eye diagram opening is thus a measure of the suitability of the FL for high bit rate modulation [24].

Figures 6(b)-(d) show simulated eye diagrams for the FM amplitude of the cross-port field, through-port power, and cross-port power of the PhC-FL with $Q=500$ and $L=5 \mu \mathrm{m}$ for $J=5 J_{\text {th }}$, in response to a 64-bit PRBS modulation of amplitude $0 \leq \delta_{\mathrm{c}} \leq 1$ (Fig. 6(a)). As can be seen, the eye diagrams for the FM amplitude are wide open, confirming the $\mathrm{THz}$ wide $\mathrm{FM}$ modulation bandwidth predicted by the SMS analysis. However, the eye diagram for the cross port power exhibits a power variation of $40 \%$ which is undesirable since we are looking for pure FM modulation without any power variation. On the other hand, the eye diagram for through-port power exhibits an open pattern, verifies its large 3-dB bandwidth which extends to $\mathrm{THz}$ frequencies and predicted by SMS analysis.

By decreasing the modulation amplitude to $0 \leq \delta_{\mathrm{c}}(\mathrm{t}) \leq 0.5$, the difference between the power levels 0 and 1 decreases, as depicted in Fig. 7(a) and (c)). Consequently, the variation of the cross-port power decreases to $11 \%$ (Fig. 7(b)), improving the purity of the signal. Optimization of the modulation properties of the laser is beyond the scope of this paper, besides, noting that we also need to take into account the demodulation techniques used for detecting the FM-modulated signal.

Finally, the cavity coupling rate and the quality factor of the nanocavity can be engineered with important consequences for

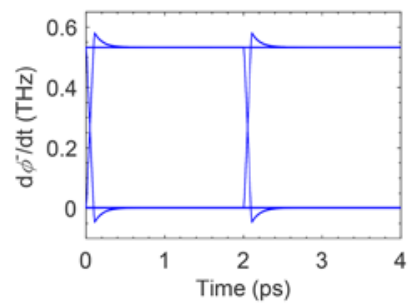

(a)

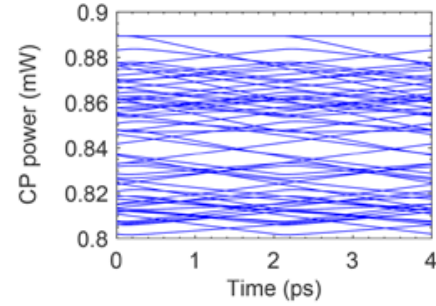

(b)

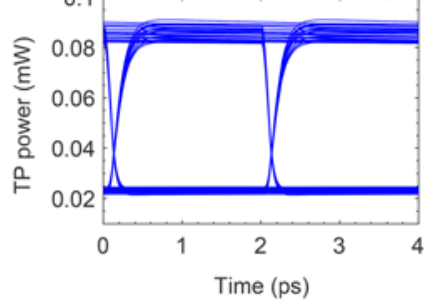

(c)

Fig. 7. Nanocavity modulation. Eye diagrams for the (a) cross-port FM amplitude, (b) cross-port power and (c) through-port power in a PhC-FL with $Q=500$ and $L=5 \mu \mathrm{m}$ for $J=5 J_{\text {th. }}$. The nanocavity detuning is modulated by an input pulse sequence similar to that in Fig. 6(a) with a modulation amplitude of 0.5 .

the laser dynamics. To investigate the effect of the nanocavity quality factor on the temporal response of the large-signal modulation of the PhC FL, we consider three different nanocavity quality factors of $Q=500,1000$, and 1500 under a fixed bias current of $J=2.3 \mathrm{~mA}$. Here we consider modulation of the nanocavity with an amplitude of $0 \leq \delta_{\mathrm{c}} \leq 0$. 5. Fig. 8(a)8(c) shows that by decreasing the quality factor of the nanocavity via an increased coupling to the waveguide, the average power increases due to the increase of the maximum Fano mirror reflectivity and the corresponding reduction of the threshold current. In addition, by increasing the quality factor of the nanocavity from 500 to 1500 , the power variation decreases from $11 \%$ to $0.8 \%$, due to the smaller absolute variation of the nanocavity resonance frequency. Thus, the power modulation variation decreases consequently, which leads to a more pure FM modulated signal. As expected from the SMS analysis, as the nanocavity quality factor increases, the 3-dB bandwidth decreases since $Q \propto \gamma_{T}^{-1}$. Correspondingly, the eye diagrams start to close (the difference between 0 and 1 levels decreases) as seen in Figs. 8(d)-(f).

\section{CONCLUSION}

In conclusion, we have studied small- and large-signal modulation properties of photonic crystal Fano lasers. In particular, conventional current modulation has been compared to the case of modulating the resonance frequency of the nanocavity governing the Fano mirror, which controls the wavelength and intensity of the laser. It was shown that the Fano laser has the prospect of being modulated at frequencies of several hundred gigahertz when considering modulation of the nanocavity. Furthermore, the effect of the nanocavity quality factor $(Q)$ on the modulation properties was investigated, showing that this provides an important design parameter. 

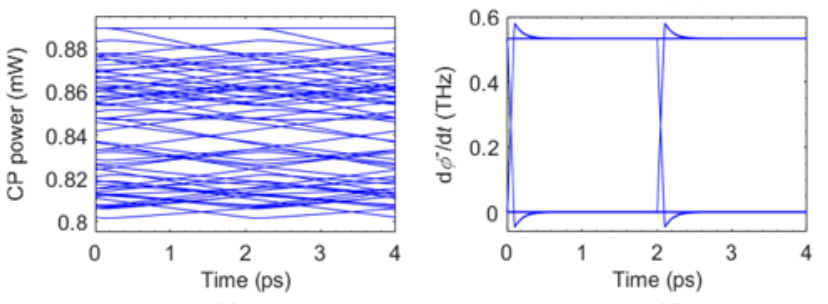

(a)

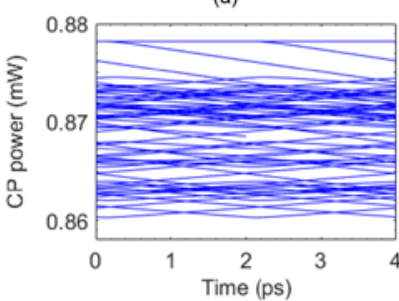

(b)

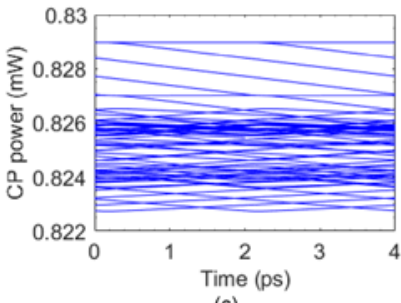

(c)

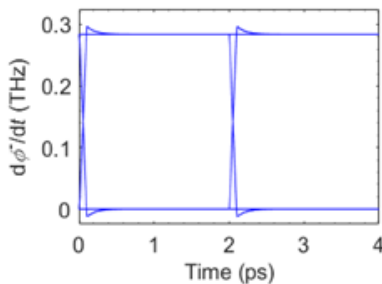

(e)

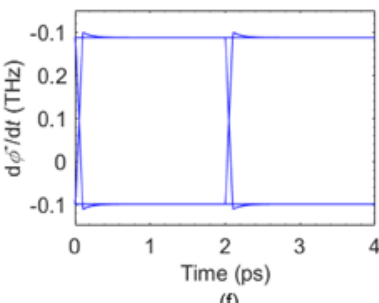

(f)

Figure 8: Nanocavity modulation. Eye pattern diagrams for the cross-port power of $\mathrm{PhC}$ FL with $L=5 \mu \mathrm{m}, J=2.3 \mathrm{~mA}$, in response to an input pulse sequence similar to that shown in Fig. 6(a) except for a for a detuning amplitude of $0 \leq \delta_{\mathrm{c}} \leq 0$. 5. The nanocavity quality factors are (a) $Q=500$, (b) 1000 , and (c) 1500 , respectively. (d)-(f) are the corresponding eye patterns for the FM amplitude for three different nanocavity quality factors of 500,1000 , and 1500 .

Future work should analyze different methods for modulating the nanocavity as well as optimizing the performance under consideration of the actual detection technique.

\section{REFERENCES}

[1] O. Painter, R. K. Lee, Axel Scherer, A. Yariv, J. D. O'brien, P. D. Dapkus, and I. Kim, "Two-dimensional photonic band-gap defect mode laser,” Science vol. 284, no. 5421, pp. 1819-1821, 1999.

[2] H.G. Park, S.H. Kim, S.H. Kwon, Y.G. Ju, J.K. Yang, J.H. Baek, S.B. Kim, and Y.H. Lee, "Electrically driven single-cell photonic crystal laser,” Science vol. 305, no. 5689, pp.1444-1447, 2004.

[3] T. Baba, D. Sano, K. Nozaki, K. Inoshita, Y. Kuroki, and F. Koyama, "Observation of fast spontaneous emission decay in GaInAsP photonic crystal point defect nanocavity at room temperature.” Applied physics letters vol. 85, no. 18, pp. 3989-3991, 2004.

[4] H. Altug, D. Englund, and J. Vučković, "Ultrafast photonic crystal nanocavity laser,” Nature physics vol. 2, no. 7, p. 484, 2006.

[5] D. Englund, H. Altug, B. Ellis, and J. Vučković, "Ultrafast photonic crystal lasers," Laser \& Photonics Reviews vol. 2, no. 4, pp. 264-274, 2008.

[6] S. Matsuo, A. Shinya, T. Kakitsuka, K. Nozaki, T. Segawa, T. Sato, Y. Kawaguchi, and M. Notomi, "High-speed ultracompact buried heterostructure photonic-crystal laser with $13 \mathrm{fJ}$ of energy consumed per bit transmitted," Nature Photonics vol. 4, no. 9, p. 648, 2010.

[7] S. Matsuo, T. Sato, K. Takeda, A. Shinya, K. Nozaki, H. Taniyama, M. Notomi, K. Hasebe, and T. Kakitsuka, "Ultralow operating energy electrically driven photonic crystal lasers," IEEE Journal of Selected Topics in Quantum Electronics vol. 19, no. 4, pp. 4900311-4900311, 2013.

[8] W. Xue, Y. Yu, L. Ottaviano, Y. Chen, E. Semenova, K. Yvind, and J. Mork, "Threshold characteristics of slow-light photonic crystal lasers,” Physical review letters vol. 116, no. 6, p. 063901, 2016.

[9] K. Kiyota, T. Kise, N. okouchi, T. Ide, and T. Baba. "Various low group velocity effects in photonic crystal line defect waveguides and their demonstration by laser oscillation," Applied physics letters vol. 88, no. 20, p. 201904, 2006.

[10] Y. Zhang, M. Khan, Y. Huang, J. Ryou, P. Deotare, R. Dupuis, and M. Lončar, "Photonic crystal nanobeam lasers," Applied physics letters vol. 97, no. 5, p. 051104, 2010.

[11] J. Mork, Y. Chen, and M. Heuck, "Photonic crystal Fano laser: terahertz modulation and ultrashort pulse generation," Physical review letters vol. 113, no. 16, p. 163901, 2014

[12] T. S. Rasmussen, Y. Yu, and J. Mork, "Modes, stability, and smallsignal response of photonic crystal Fano lasers," Optics express, vol. 26, no. 13, pp.16365-16376, 2018.

[13] Y. Yu, W. Xue, E. Semenova, K. Yvind, and J. Mork, "Demonstration of a self-pulsing photonic crystal Fano laser,” Nature Photonics vol. 11, no. 2, p. 81, 2017.

[14] T. S. Rasmussen, Y. Yu, and J. Mork, "Theory of Self-pulsing in Photonic Crystal Fano Lasers,” Laser \& Photonics Reviews vol. 11, no. 5, p. 17000892017.

[15] J. D. Joannopoulos, S. G. Johnson, J. N. Winn, and R. D. Meade, Photonic crystals: molding the flow of light. Princeton university press, 2011.

[16] S. Fan, "Sharp asymmetric line shapes in side-coupled waveguide-cavity systems," Applied physics letters vol. 80, no. 6, pp. 908-910, 2002.

[17] L. A. Coldren, S. W. Corzine, and M. L. Mashanovitch, Diode lasers and photonic integrated circuits. Vol. 218. John Wiley \& Sons, 2012.

[18] W.Xue, L. Ottaviano, Y. Chen, E. Semenova, Y. Yu, A. Lupi, J.Mork, and K. Yvind. "Thermal analysis of line-defect photonic crystal lasers," Optics express, vol. 23, no. 14 pp. 18277-18287, 2015.

[19] L. D. Haret, X. Checoury, F. Bayle, N. Cazier, P. Boucaud, S. Combrié, and A. de Rossi, "Schottky MSM junctions for carrier depletion in silicon photonic crystal microcavities," Optics express vol. 21, no. 8, pp. 10324-10334, 2013.

[20] A. F. Koenderink, M. Kafesaki, B. C. Buchler, and V. Sandoghdar, "Controlling the resonance of a photonic crystal microcavity by a nearfield probe,” Physical review letters vol. 95, no. 15, p. 153904, 2005.

[21] M. Heuck, P. T. Kristensen, Y. Elesin, and J. Mørk, "Improved switching using Fano resonances in photonic crystal structures," Optics letters vol. 38, no. 14, pp. 2466-2468, 2013.

[22] Y. Yu, E. Palushani, M. Heuck, N. Kuznetsova, P. T. Kristensen, S. Ek, D. Vukovic, C. Peucheret, L.K. Oxenløwe, S. Combrié, and A. de Rossi, "Switching characteristics of an InP photonic crystal nanocavity: Experiment and theory," Optics express vol. 21, no. 25 pp. 31047-31061, 2013.

[23] T. Tanabe, M. Notomi, H. Taniyama, and E. Kuramochi, "Dynamic release of trapped light from an ultrahigh-Q nanocavity via adiabatic frequency tuning," Physical review letters vol. 102, no. 4, p. 043907 , 2009.

[24] K. Lüdge, M.J Bormann, E. Malić, P. Hövel, M. Kuntz, D. Bimberg, A Knorr, and E. Schöll, "Turn-on dynamics and modulation response in semiconductor quantum dot lasers,” Physical Review B vol. 78, no. 3 p. 035316, 2008

Aref Rasoulzadeh Zali was born in Tabriz, Iran in 1986. He received the B. Sc. and the M. A. degrees in Electrical engineering from Tabriz University, Tabriz, Iran, in 2010, and Tarbiat Modares University, Tehran, Iran, in 2012, respectively. He is currently working on his $\mathrm{PhD}$ project. His research interests include photonic devices such as photodetectors, lasers, and SOAs.

Mohammad Kazem Moravvej-Farshi (2005 SM) received the B. Sc. and the M. A. degrees in physics from Sharif University of Technology (SUT), Tehran, Iran, in 1976, and the University of Southern California (USC), Los Angeles, California, in 1978, respectively, the M. Sc. and the Ph. D degrees in 
electronics from the University of California at Santa Barbara (UCSB), in 1980, and the University of New South Wales (UNSW), Sydney, Australia, in 1987, respectively. From 1980 to 1984, he was a member of research staff with the Division of Microwave, Iran Telecommunication Research Center (ITRC). He joined Tarbiat Modares University (TMU) in 1987, where he is currently a Professor of Electronics and head of Nano Plasmo-Photonic Research Group.

Professor Moravvej Farshi is currently a senior member of IEEE and a senior member of the Optical Society of America (OSA). He is also one of the founders of the Optics and Photonics Society of Iran.

Yi Yu received the B.Sc. and M.Eng. degrees from Huazhong University of Science and Technology, China, in 2008 and 2011, respectively, and his Ph.D. degree from Technical University of Denmark (DTU), Denmark, in 2015. Since then he worked as a Postdoc at DTU Fotonik and now at the Institute of
Physics in École Polytechnique Fédérale de Lausanne, Switzerland. His current research focuses on nano-cavity lasers and quantum nanophotonic structures.

Jesper Mørk received the M.Sc., Ph.D., and Dr. Techn. degrees from the Technical University of Denmark (DTU), Lyngby, in 1986, 1988, and 2003, respectively. Since 2002 he is a Professor in semiconductor photonics and since 2008 he is heading the Nanophotonics Section at DTU Fotonik, Technical University of Denmark. He is the author of more than 245 papers in refereed journals and around 350 contributions to international conferences, including more than 80 invited talks. His current research interests include semiconductor quantum photonics, photonic crystal structures, slow light, nano- and micro-cavity lasers and integrated photonics. Jesper Mørk is a Fellow of OSA and serves as Associate Editor of Optica. 